\title{
Die Sprache der Wissenschaft
}

Thema meine Beitrags ist nicht die Sprache meines Faches, das wäre kurz abgehandelt, sondern in der Tat die Sprache der Wissenschaften. Ich möchte mit Ihnen, um mein Ziel zu erreichen, sechs Schritte durchgehen, um am Ende ein „pluralistisches Weltmodell“ zu offerieren. Etwas Bescheideneres kann ich nicht bieten.

\section{(1.) Wissen und Sprache}

Herr Trabant hat es bereits anklingen lassen, hier wird eine grundlegende Weichenstellung vollzogen, die letztlich entscheidet, ob unser Thema nur ein Randphänomen der Wissenschaft ist oder den Kern der Wissenschaft betrifft. Das alte Modell, das auf Platon und Aristoteles zurückgeht und von Descartes dann noch einmal bekräftigt worden ist, lautet - und einige haben es ja heute schon vertreten ohne es vielleicht zu bemerken -: dass die Sprache dem Wissen äußerlich ist, es nur "ausdrückt". Die dahinter stehende Vorstellung ist, um es metaphorisch auszudrücken, dass die Sprache auf der Vorderbühne spielt, aber die eigentlichen Entscheidungen auf der Hinterbühne des Wissens fallen. Diese Vorstellung wird oft mit dem Kategorienpaar von „Aus-druck - Erleben” verbunden: die Welt des Erlebens ist die eigentliche (Innen-) Welt, von der aus gelegentlich Nachrichten nach Außen "gedrückt" werden. Die Philosophen nennen dieses Modell des Verhältnisses von Wissen und Sprache "Mentalismus". Es gibt starke Argumente, den Mentalismus abzulehnen und damit einem Anti-Mentalismus beizutreten. Abgesehen davon, dass es auch schon bei den Griechen Vorformen gab, ist der Anti-Mentalismus im wesentlichen durch die großen Drei der deutschen Sprachphilosophie Hamann, Herder und Humboldt vertreten worden, im 20. Jahrhundert ist diese Position dann von Heidegger, Wittgenstein, Austin und anderen noch einmal unterstrichen und verfeinert worden. Die These des Anti-Mentalismus ist also: Wissen ist von vorneherein sprachlich verfasst. Hierzu muss man sich allerdings - um diese These gegen offene Scheunentore von Einwänden zu schützen -zwei Präsuppositionen klar machen. 
Erstens: Man darf sprachliche Manifestationen nicht nur am Modus der phonetischen Verlautbarung festmachen. Sprache kann unhörbar sein, sie kann nicht nur Rede, sondern auch "Schreibe" oder auch "Zeige" sein, d.h. sich nicht nur phonetisch, sondern auch graphisch und gestisch manifestieren, Die Funktionalität der Taubstummensprache ist ein Beleg für den Fall der gestischen Manifestation; und Amerika-Reisende fällt auf, dass dort alle phonetischen Wortsendungen im Fernsehen aufgrund eines Diskriminierungsverbots immer auch in gestischer Sprache dargestellt sein müssen. Also lösen wir uns von der Unterstellung, Sprache müsse immer etwas Lautes oder etwas Verlautetes sein.

Zweitens dürfen wir Sprache nicht nur - wie es in weiten Bereichen der Kommunikationswissenschaften und Linguistik geschieht - als ein Signalsystem verstehen. Signale sind nämlich nichts anderes als gewissermaßen äußerliche Momente dahinter stehender Handlungen (wie Behauptungen, Aufforderungen, Versprechen usw.). Gerade dieser performative Hintergrund der Sprache gerät im Signalmodell leicht in Vergessenheit: im allgemeinen reden wir (Ausnahmen sind etwa das Testen einer Mikrophon-Anlage) um durch die Äußerung etwas zu tun. Sprache ist so etwas wie ein strukturiertes Ensemble von Rede- (Schreibe-, Zeige) Handlungen. Das wichtigste Argument für diese Sicht der Dinge ist, dass, wenn wir mentale Vorgänge, also vorgeblich sprachloses Wissen oder sprachfreies Denken, beschreiben wollen, immer auf sprachliche Manifestationen rekurrieren müssen. Was ein Urteil ist, explizieren wir, indem wir Behauptungen studieren. Was ist ein Begriff ist, explizieren wir, indem wir Prädikatoren studieren. Was richtiges Denken ist, studieren wir, indem wir richtiges Argumentieren untersuchen. Vorgebliche Denkgesetze sind nur als Argumentationsregeln fassbar und mitteilbar. Das heißt, wer zwischen einem sprachlosen Wissen einerseits und der dieses Wissen „aus-drückenden" Sprache andererseits unterscheidet, vollzieht eine überflüssige Wirklichkeitsverdoppelung. Sprachliche Kommunikation besteht somit nicht nur im Senden und Empfangen von Signalen, sondern sprachliche Kommunikation ist ein regelgeleiteter Austausch von sprachlichen Handlungen zwischen Rede-Akteuren. In der wissenschaftlichen Kommunikation nehmen die Akteure die Rollen von Proponenten und Opponenten ein, die bestimmte Dinge nach Regeln tun. Solche Redehandlungsregeln steuern einen Komplex wechselseitiger Berechtigungen und Verpflichtungen, die beachten muss, wer aussichtsreich an wissenschaftlicher Kommunikation teilnehmen will. Daher muss das Instrumentarium der Sprachrekonstruktion die Erfassung solcher Regeln überhaupt vorsehen und das ist beim Signalmodell der Kommunikation nicht gegeben. Regeln kann man verletzen, und 
wenn man sie verletzt, schlägt der Kommunikationsversuch fehl. Es gibt demgemäß durchaus komplizierte Regelgefüge, die einzuhalten sind, wenn wir Kommunikationsversuche als gelungen oder nicht-gelungen beurteilen. Das Signal-Modell hat neben der Wirklichkeitsverdopplung und der Blindheit für den performativen Charakter der Sprache einen weiteren negativen Effekt, es lenkt den Blick vor allem auf die scheinbar elementaren Signale der Sprache, nämlich die Wörter. Das hat wiederum die irreführende Folge, dass die Debatte über Wissenschaftssprachen vor allem als Debatte über Lexika und deren Übersetzbarkeit geführt. wird

\section{(2.) Sprache und Sprachen}

Ich spreche bisher über die Sprache, was vielen unter den Zuhörern vielleicht suggeriert, ich nähme an, es gäbe so etwas wie eine singuläre Menschheitssprache. Das ist natürlich ein Missverständnis. Vielmehr deutet die Verwendung des generischen Singulars "die" Sprache an, dass hier eine Abstraktion unterstellt wird, so wie wir auch über "den" Menschen, "den" Staat, "das" Recht usw. sprechen. Die generische Verwendung von Wörtern hat eine unverzichtbare abstraktive Funktion, die für unser Thema etwas zu erläutern ist. Tatsächlich manifestiert sich die Sprache in einer Pluralität von Sprachen, und anders ist sie nicht zu haben. Man spricht von "natürlichen" Sprachen, mir liegt näher, von „positiven" Sprachen zu sprechen, so wie man vom „positiven" Recht spricht. Und von solchen positiven Sprachen gibt es bei großzügiger Zählung etliche 1000 auf der Welt, wenn man ein gewisses Größenkriterium einführt, vielleicht 150 bis 200. Das sind die Sprachen, mit denen wir uns hier auseinanderzusetzen haben, denn mit jeder von ihnen kann sicher prinzipiell wissenschaftliche Kommunikation stattfinden. Da wir die Sprachen somit unter einem Zweckgesichtspunkt untersuchen, können wir sie unter dem Gesichtspunkt ihrer instrumentellen Gleichheit betrachten und in diesem Sinne von "der" Sprache sprechen. „Die” Wissenschaftssprache ist das Ensemble aller Wissenschaftssprachen, betrachtet unter dem Gesichtspunkt ihrer instrumentellen Gleichheit.

\section{(3.) Die Instrumentalität der Sprache}

Somit ist mit der Rede von "der" Sprache implizit die Instrumentalität der Sprachen unterstellt. Wenn wir über Wissenschaft und Sprache sprechen, fragen wir uns ja vor allem auch, welche Sprache oder in welcher Façon Sprache sich für Wissenschaft eignet. Reden, das heißt, die Exekution 
von Sprache, ist in vielen Kontexten Mittel zum Zweck. Dieser Zweck ist primär der Vollzug wissenschaftlicher Kommunikation zwischen Proponenten und Opponenten, und erst in Abhängigkeit davon auch sekundär, einen Sachverhalt zu repräsentieren. Wenn etwas einem Zweck dienen soll, ist die Frage der (besseren oder schlechteren) Eignung zu stellen. Fragen wir uns also, welche Sprache sich für jeden Zweck eignet. Wenn man diesbezüglich die erwähnten 150 bis 200 Kandidaten betrachtet, ist es allerdings sehr unwahrscheinlich, dass sich jede Sprache instrumentell für jeden Zweck gleich gut eignet. Das wird man auch bei anderen instrumentellen Fragen für unwahrscheinlich halten. Eignen sich alle Wagenheber für jeden Zweck gleich gut? Kindersitze, Gartenstauden, Wörterbücher, Professoren, Dirigenten - das wäre doch eine merkwürdiger Zufall, wenn alle für alles gleich gut geeignet wären. Vielmehr wird man annehmen dürfen, dass sich Manches für Manches besser und für Manches schlechter eignet. Instrumentelle Differenzierung ist im Rahmen der Zweck-Mittel-Rationalität der Normalfall. Deswegen ist auch sicher nichts Obszönes an der These, Griechisch und Deutsch seien für die Aufgaben der Philosophie besser geeignet als Lateinisch und Englisch. Das hat Heidegger behauptet, und vielleicht hat er recht oder unrecht - das muss man empirisch überprüfen - aber durch die Aufstellung dieser These bekundet er nicht irgendeine Nähe zum Nationalsozialismus, zumal er ja nicht Deutsch gesagt hat, sondern Griechisch und Deutsch. Und so könnte es auch sein, so etwas liest man gelegentlich, dass sich das Lateinische besonders gut für Zwecke des Rechts, das Französische für Zwecke des Dramas, das Englische für Zwecke der Seefahrt, das Grönländische für die Aufklärung von Verbrechen im Schnee und das Arabische für die Pferdezucht eigneten. Alles das sind wahre/falsche Aussagen, die Sprachwissenschaftler überprüfen mögen. Und deswegen ist die Festlegung einer Sprache der Wissenschaft - und damit ein gewisser Sprachimperialismus - schon wegen der großen Zahl der Sprachen, etliche 1000, und der großen Zahl der Fächer, der Hochschulverband zählt etliche 1000, allein aufgrund der Permutativität von Sprachen und Fächern ein Wahnsinnsunternehmen. Dass es die Sprache der Wissenschaft gibt - diese Vorstellung sollte man fahren lassen.

\section{(4.) Eine Sprache beherrschen}

Eine Sprache beherrschen bedeutet weitaus mehr, ich wiederhole das, als das Verfügen über ein Lexikon. Wäre nur das Lexikon das Problem, bräuchten wir die Debatte gar nicht zu führen, denn es gibt ein internationales wissenschaftslexikalisches Esperanto, das über alle Diszipli- 
nen hinweg zu 90 Prozent identisch ist. Dieses Lexikon ist im übrigen nicht englisch, sondern ein griechisch-lateinisches Hybrid. "Science", „isomorph", „äquivalent”, „Modell“, „Exponent”, „Gravitation“, „Hexafluorphosphat", „Eukaryonten", „Genom”, "Leukämie”, „Computer" „Partizipation”, „System”, „Bruttosozialprodukt”, „Rezeptionsästhetik", das ist alles Graeco-Latein. Es gibt ganz wenige Ausnahmen: "Ensemble", "surjectif" sagen die Mathematiker, das ist französisch; „Eigenwert, Gestalt”, „Realpolitik“, das ist deutsch; „onbetrouwbaarheid" ist niederländisch und "Ombudsmann" ist schwedisch; Es gibt also ein paar nicht-griechisch-lateinische Wörter in den Wissenschaftssprachen. Das Sprachproblem ist also grob gesagt keines des Lexikons der Sprache, sondern eines der semantischen und pragmatischen Regeln. Zu den Gelingensbedingungen für sprachliche Handlungen gehört unter anderem auch das Beherrschen von Untertönen, die Beherrschung von Sprachebenen-Problemen, das Verstehen von Sprachwitzen, des Kennen der Bildungsregeln für Neologismen und dergleichen. Zum Beispiel Untertöne: "collective authorship", da denkt der Deutsche, aber nicht der Engländer, ein wenig an DDR-Publikationen. „Big collaboration“, da denkt der Franzose und der Deutsche, aber nicht der Engländer, ein wenig an Verrat; "Euthanasia“, da denkt der Deutsche ein wenig an Verbrechen der Nazis, aber sonst niemand. Ein Beispiel für SprachebenenProbleme: Die im Englischen so routinierten Naturwissenschaftler übersetzen doch mal bitte bis zum Ende meines Vortrages den Satz: „Diese ausgelutschte Hypothese noch mal aufzuwärmen, wäre doch bescheuert." Aber bitte: Versuchen Sie die sprachebenen-adäquate Übersetzung (nur das Wort "Hypothese" lexikalisch zu übersetzen, das kann es nicht sein). Ein Beispiel für Sprachwitz oder Sprachpointen: Das RheinlandPfälzische Wissenschaftsministerium wirbt mit folgendem Satz: „Wissenschaft ist die gesellschaftliche Institution, die Wissen schafft." Das geht mit "science" einfach nicht. Ein Beispiel für Neologismen: Wir versuchen gelegentlich, neue Wörter einzuführen für neue Sachverhalte, wie man dann sagt; Heidegger glaubte behaupten zu sollen: Bevor ein Gegenstand für die Wissenschaft "vorhanden" ist, muss er in der alltäglichen Erfahrung "zuhanden" sein. Ich nehme an, das versteht jeder, der deutsch spricht. Wie übersetzen wir das ins Englische? Es gibt drei Übersetzungen von Sein und Zeit durch native speaker - wohlgemerkt. Hofstadter übersetzt "zuhanden" mit "being handy" - übrigens ist damit das Gerücht widerlegt, "handy" gäbe es im Englischen gar nicht - und „Vorhanden" mit "extant" (eine vornehme, hochsprachliche Verballhornung von "existent"). Die Hand-Metaphorik, die im Deutschen leitend ist, wird im Englischen somit aufgegeben. „Existenz" heißt bei Heidegger aber eigentlich ganz etwas anderes, die Übersetzung ist sogar her- 
meneutisch gefährlich. Macquarrie und Robinson übersetzen „zuhanden" mit "ready to hand" und "vorhanden" mit "present-at-hand". Die Hand-Metaphorik wird also beibehalten, aber leider bringt das Wort „present" einen Zeitmodus mit ins Spiel, der bei „zuhanden” gar nicht anklingt. Also erhalten wir eine semantische Erschleichung von Temporalität. Stambaugh (in der jüngsten Übertragung) übersetzt „zuhanden" mit "being at hand", "vorhanden" mit "objectively present", die Hand-Metaphorik wird wieder aufgegeben, statt dessen der Begriff der Objektivität eingeführt. Ein sprachlicher Missgriff, denn „Objektivität” heißt bei Heidegger etwas völlig anderes als „vorhanden sein". Solche Übersetzungsprobleme sind in jedem Fach in mehr oder weniger großer Zahl auffindbar, und die löst man nur, wenn man nicht nur denotative, sondern auch konnotative, z.B. auch metaphorische Probleme löst. Übrigens hat das mit der Erfahrung zu tun, die sicher jeder schon einmal gemacht hat, der einen eigenen Text in fremder Übersetzung liest - und das gilt nicht nur für das Englische, sondern auch für andere Sprachen -, dass man nämlich denkt: Naja, das stimmt zwar einigermaßen, aber auch irgendwie ist auch der Witz weg.

\section{(5.) Einige empirische Hypothesen}

Durch die folgenden Thesen überschreite ich die Grenzen meiner sprachphilosophischen Zuständigkeit und bitte die empirischen Sprachwissenschaftler, die folgenden Thesen kritisch zu prüfen. Bis auf weiteres erscheinen sie mir jedoch von unabweisbarer Evidenz.

Erstens: Wer eine Sprache beherrscht, verfügt in erster Linie über ein "Know how" und nicht über ein „Know that". Die Sprache des Erstsprachenerwerbs lernt man nicht durch Grammatikunterricht, sondern durch Einsozialisation, und der Lernprozess hat in der Tat, wie Wittgenstein sagt, etwas zu tun mit dem Abrichten, weil dabei ja Belohnungsund Strafmechanismen eine bedeutende Rolle spielen.

Zweitens: Die Einsozialisationsphase des Erstsprachenerwerbs endet nach der frühen Jugend, und wenn diese abgeschlossen ist, lernt man weitere Sprachen eher im Sinne des "Know that", also durch Grammatikunterricht. Echte Bi- oder gar Multi-Lingualität scheint selten, vielleicht gibt es sie gar nicht.

Drittens: Die Sprachkompetenz in einer Sprache des Zweiterwerbs ist nach allen Dimensionen gegenüber Lexikon, Konnotationen, Sprachstil, 
Sprachschichtenzuordnung, Neologismen usw. gegenüber der Sprache des ersten Erwerbs eingeschränkt.

Viertens: Die Auswahl einer Wissenschaftssprache bringt für fast alle, für die diese nicht die Sprache des Ersterwerbs ist, mehr oder weniger erhebliche kommunikative Beeinträchtigungen mit sich.

Fünftens: Das Kompetenzgefälle zwischen einer Sprache des Ersterwerbs und einer Sprache des Zweiterwerbs ist vor allem im Bereich der aktiven Sprachkompetenz, weniger im Bereich der rezeptiven Sprachkompetenz ausgeprägt.

\section{(6) Ein pluralistische Weltmodell}

Ich unterstelle zunächst, dass aus rein kulturgeschichtlich-faktischen Gründen nicht alle 150 bis 200 Großsprachen der Welt eine Affinität zu dem haben, was in der Wissenschaft geschieht. Eher wird man 6 bis 8 Sprachen faktisch-historische (nicht grundsätzlich) als besonders wissenschaftsaffin auszeichnen dürfen. Ich stelle mir also vor, dass man die Probleme der kommunikativen Beeinträchtigung für all diejenigen, für die die Sprache ihres Faches nicht die Sprache des Ersterwerbs ist, erheblich einschränken kann, wenn man annehmen darf, dass jeder Wissenschaftler bezüglich dieser 6 bis 8 Sprachen rezeptiv kompetent ist - er versteht diese Sprachen - und aktiv eine wirklich beherrscht. 